\title{
Variação sazonal dos fluxos de energia e evapotranspiração em caatinga no agreste pernambucano
} \author{
Eduardo Soares de Souza ${ }^{5}$ \\ ${ }^{1}$ Professor Associado, UAG/UFRPE, Garanhuns, PE, romualdo.lima@ufrpe.br \\ ${ }^{2}$ Estudante de Agronomia, UAG/UFRPE, Garanhuns, PE, cassio.agronomo@yahoo.com \\ ${ }^{3}$ Estudante de Agronomia, UAG/UFRPE, Garanhuns, PE, amos.barbosa@outlook.com \\ ${ }^{4}$ Professor Titular, DEN/UFPE, Recife, PE, acdantonino@gmail.com \\ ${ }^{5}$ Professor Associado, UAST/UFRPE, Serra Talhada, PE, edu_souza_pe@yahoo.com.br \\ ${ }^{(*)}$ Autor para correspondência
}

José Romualdo de Sousa Lima ${ }^{1\left(^{*}\right)}$, Cássio Lopes de Oliveira ${ }^{2}$, Amós da Cruz Barbosa ${ }^{3}$, Antonio Celso Dantas Antonino ${ }^{4}$,

\section{INFORMAÇÕES}

História do artigo:

Recebido em 16 de Junho de 2017

Aceito em 10 de agosto de 2017

Termos para indexação:

semiárido

mudanças climáticas

umidade do solo

\section{RESUMO}

Os fluxos de energia, quais sejam o saldo de radiação (Rn), os fluxos de calor latente (LE), sensível (H) e no solo (G), e a evapotranspiração (ET) têm uma grande importância em diversos aspectos do tempo e do clima e estão diretamente ligados com os fluxos de carbono. O método da correlação dos turbilhões é um dos mais usados para se medir esses fluxos, no entanto ainda é caro. Assim, o ideal é se usar um método mais barato e também preciso, como o da razão de Bowen. Desse modo, o objetivo do trabalho foi avaliar a variação sazonal de Rn, LE, H, G e ET em área de Caatinga preservada, localizada no município de São João, PE, usando o método da razão de Bowen. Para tal, instalou-se uma torre de $8 \mathrm{~m}$ de altura no centro da área, contendo um pluviógrafo, um saldo radiômetro e sensores para medida da temperatura e da umidade relativa do ar, em dois níveis acima do dossel da Caatinga; além disso, foram instalados sensor de umidade do solo e um fluxímetro, a 5,0 cm de profundidade. Verificou-se que a maior parte do Rn foi usada no H e foi controlada pela variação sazonal da precipitação pluvial e da umidade volumétrica do solo. A ET total (852 mm) e média (2,3 $\left.\mathrm{mm} \mathrm{d}^{-1}\right)$ da Caatinga apresentou valores elevados, em decorrência da boa disponibilidade de água no solo no período estudado.

(c) 2017 SBAgro. Todos os direitos reservados.

\section{Introdução}

A Caatinga é o único bioma exclusivamente brasileiro, ocupando uma área de aproximadamente 1 milhão de $\mathrm{km}^{2}$, com uma população de mais de 20 milhões de habitantes. As chuvas na região são baixas $(400-800 \mathrm{~mm})$ e com elevada variabilidade temporal e espacial, com temperaturas altas e elevada evapotranspiração potencial, causando déficit hídrico na maior parte do ano (MENEZES et al., 2012).

Nessa região, a degradação dos recursos naturais tem sido provocada pelo aumento da intensidade de uso do solo e redução da cobertura vegetal nativa (SAMPAIO et al., 2004). A retirada da Caatinga, comumente é realizada para a implantação de pastos e culturas agrícolas, o que, junta- 
mente com a elevada variabilidade climática, pode provocar degradação do solo, contribuindo para o aumento das emissões de $\mathrm{CO}_{2}$ para a atmosfera. Ademais dos problemas oriundos da degradação ambiental, o cenário de mudanças climáticas para o Semiárido brasileiro, prevê, conforme Cirilo (2008), elevação da temperatura, tendo como consequência aumento da evaporação nos corpos d'água, redução da recarga dos aquíferos em até 70\% até o ano 2050.

De acordo com Souza et al. (2015a), uma das formas de avaliar as respostas dos ecossistemas às condições ambientais é por meio do balanço de energia, representado pela sua partição nos fluxos de calor latente (LE), de calor sensível (H) e de calor no solo (G). Diversos processos importantes que ocorrem nos ecossistemas, como a fotossíntese e a produtividade, estão associados com os fluxos LE e H (WEVER et al., 2002). Além disso, os estudos desses fluxos são importantes, pois possibilitam identificar intef rações existentes entre elas, além de informações sobre a quantidade de água perdida para a atmosfera (OLIVEIRA et al., 2006), ou seja, evapotranspiração (ET). De acordo com Barreto et al. (2009) em algumas regiões, a ET de uma bacia hidrográfica representa parcela superior à água que escoa superficialmente.

O método da covariância dos vórtices turbulentos é um método que vem sendo utilizado para quantificar os fluxos de energia e ET dos ecossistemas (BALDOCCHI, 2003), no entanto ainda é um método caro, devido ao alto valor dos instrumentos. Outro método usado para se medir os fluxos de energia e a ET é o da razão de Bowen, que tem como principais vantagens: medições simples e diretas; não requer informações a respeito das características aerodinâmicas da superfície de interesse; poder integrar fluxos de calor latente sobre grandes áreas; nas suas desvantagens se incluem a sensibilidade às predisposições dos instrumentos que medem os gradientes e os componentes do balanço de energia; a possibilidade de descontinuidade de dados quando a razão de Bowen se aproxima de -1 (LIMA et al., 2005; LIMA et al., 2011; TODD et al., 2000).

O estudo dos fluxos de energia e/ou da ET em área de Caatinga já foi realizado em trabalhos prévios (BORGES et al., 2016; OLIVEIRA et al., 2006; SOUZA et al., 2015a; TEIXEIRA et al., 2008). No entanto, todas essas pesquisas usaram o método da covariância dos vórtices turbulentos. Além disso, todas essas pesquisas foram realizadas em ambiente semiárido, sendo a maior parte delas com restrição hídrica, o que tem grande influência na partição dos componentes do balanço de energia e na ET. Pelo nosso conhecimento, até o momento, essa é a primeira pesquisa que mediu os fluxos de energia e a ET em Caatinga no agreste pernambucano, que é uma região que possui uma melhor disponibilidade hídrica.

Desse modo, o objetivo do presente trabalho foi avaliar a variação sazonal dos fluxos de energia e a ET em área de Caatinga preservada no agreste pernambucano, usando o método da razão de Bowen. Nossa hipótese é que, mesmo na região agreste, a disponibilidade de água no solo e a variação da precipitação pluvial irão controlar as variações sazonais dos fluxos de energia e da ET, bem como a partição do saldo de radiação nos fluxos de calor latente e sensível.

\section{Material e métodos}

O experimento foi realizado no período de 01 de janeiro a 31 de dezembro de 2014, numa área de 4 ha de Caatinga preservada, localizada na Fazenda Riacho do Papagaio, município de São João - PE (8 52' 30” S, 36 22' $00^{\prime \prime}$ O e 705 $\mathrm{m})$, na microrregião do Agreste Meridional, bacia hidrográfica do Rio Mundaú. A precipitação média anual é cerca de $782 \mathrm{~mm}$, sendo o quadrimestre mais chuvoso constituído dos meses de maio a agosto (APAC, 2016). o solo da área é classificado como Neossolo Regolítico (SANTOS et al., 2012).

Essa Caatinga tem uma vegetação composta dos estratos herbáceo, arbustivo e arbóreo, com altura máxima de 6 $m$, sendo as espécies botânicas mais comumente encontradas: Commiphora leptophloeos (Mart.) J.B. Gillett, Cynophalla flexuosa (L.) J. Presl., Erythroxylum SP, Guapira cf. laxa (Netto) Furlan, Ziziphus joazeiro Mart., Croton Blanchetianus, Croton Heliotropiifolius, Mimosa tenuiflora (Willd.) Poir.

Para a determinação dos fluxos de energia e da ET foi instalada uma torre micrometeorológica de $8 \mathrm{~m}$ de altura no centro da área. Essa torre continha dois sensores de medidas da temperatura e da umidade relativa do ar (modelo HMP45C, Vaisala, Campbell Scientific Inc., Logan, UT, USA), em dois níveis ( $\mathrm{z} 1=1 \mathrm{~m}$ e z2 = $2 \mathrm{~m}$ ) acima do dossel da vegetação; além desses sensores, instalou-se um piranômetro (modelo LI-200X, LI-COR Inc., Lincoln, NE, USA) para a medida da radiação global (Rg), um saldo radiômetro (modelo NRLITE, Kipp \& Zonen, Delft, Netherlands) para a medição do saldo de radiação (Rn) e um pluviógrafo (modelo TE 525 WS-L, Texas Electronics, Dallas, TX, USA), para a medida da precipitação pluvial. Para a medida do fluxo de calor no solo foi instalado um fluxímetro (modelo HFT3, REBS, Seattle, WA, USA) na profundidade $\mathrm{z}=0,05 \mathrm{~m}$, juntamente com um sensor de umidade do solo (modelo CS616, Campbell Scientific Inc., Logan, UT, USA), na mesma profundidade.

O balanço de energia é descrito pela equação 1 (LIMA et al., 2013):

$$
\mathrm{Rn}=\mathrm{G}+\mathrm{H}+\mathrm{LE}
$$

sendo $\mathrm{Rn}$ o saldo de radiação $\left(\mathrm{W} \mathrm{m}^{-2}\right), \mathrm{G}$ o fluxo de calor no solo $\left(\mathrm{W} \mathrm{m}^{-2}\right)$, H o fluxo de calor sensível $\left(\mathrm{W} \mathrm{m}^{-2}\right)$ e LE o fluxo de calor latente $\left(\mathrm{W} \mathrm{m}^{-2}\right)$. 
A partição da energia disponível (Rn-G) entre LE e H, pode ser obtida pelo método do balanço de energia, baseado na razão de Bowen $(\beta)$, obtida com as diferenças na temperatura do ar e na pressão de vapor, em dois níveis (LIMA et al., 2013):

$$
\beta=\frac{H}{L E}=\gamma \frac{\Delta T}{\Delta e}
$$

em que $\gamma$ é a constante psicrométrica $\left(0,066 \mathrm{kPa}{ }^{\circ} \mathrm{C}^{-1}\right) ; \Delta \mathrm{T}$ a diferença de temperatura do ar $\left({ }^{\circ} \mathrm{C}\right)$ e $\Delta \mathrm{e}$ a diferença da pressão de vapor $(\mathrm{kPa})$.

A partir das medições do saldo de radiação (Rn), do fluxo de calor no solo $(\mathrm{G})$ e das diferenças de temperatura do ar (DT) e da pressão de vapor (De), juntamente com a equação simplificada do balanço de energia (Eq. 1) e se utilizando a razão de Bowen ( $\beta$ ) (Eq. 2), procedeu-se ao cálculo dos fluxos de calor latente (LE) e calor sensível $(\mathrm{H})$ segundo as equações:

$$
\begin{aligned}
& \mathrm{LE}=\frac{\mathrm{Rn}-\mathrm{G}}{1+\beta} \\
& \mathrm{H}=\frac{\beta}{1+\beta}(\mathrm{Rn}-\mathrm{G})
\end{aligned}
$$

A evapotranspiração ( $\mathrm{mm}$ ) foi obtida dividindo-se o LE $\left(\mathrm{MJ} \mathrm{m}^{-2} \mathrm{~d}^{-1}\right)$ pelo calor latente de vaporização, considerado como constante $\left(2,45 \mathrm{MJ} \mathrm{kg}^{-1}\right)$. Os valores diários de ET foram obtidos somando-se todos os valores de cada trinta minutos para um período de 24 horas.

\section{Resultados e discussão}

Foram registrados para o período de 01 de Janeiro a 31 de Dezembro de 2014, 754,3 mm de precipitação pluvial, ou seja, 96,5\% da média anual histórica para a precipitação (782,0 mm). Além disso, desse total pluviométrico, 43,8\% ocorreram entre os meses de Maio a Agosto (Figura 1), confirmando que o quadrimestre mais chuvoso dessa região ocorre nesse período. Observa-se, ainda, que dos 365 dias estudados, ocorreram chuvas em 182 dias (49,8\%), sendo que em somente 21 dias as chuvas foram acima de $10 \mathrm{~mm}$, com os maiores eventos nos meses de abril (dias 05 e 06, com 45,6 e 47,1 mm, respectivamente) e de dezembro (dia 05/12 com 52,6 mm). Esses três eventos de chuva corresponderam a 145,3 $\mathrm{mm}$ (19,3\% do total de chuvas).

Quanto a umidade do solo (Figura 1) notou-se que no período de Janeiro a Março de 2014 seus valores são pequenos, oscilando entre 0,01 e $0,02 \mathrm{~m}^{3} \mathrm{~m}^{-3}$. A partir de Abril de 2014, com o aumento das precipitações, os valores de umidade do solo aumentam para 0,04-0,05 $\mathrm{m}^{3} \mathrm{~m}^{-3}$, sendo que os maiores valores $\left(0,08\right.$ a $\left.0,10 \mathrm{~m}^{3} \mathrm{~m}^{-3}\right)$ ocorrem no período mais chuvoso, nos meses de Junho e Julho. Dessa forma, pode-se afirmar que a umidade volumétrica do solo seguiu os padrões da variação sazonal da precipitação pluvial. Os pequenos valores de umidade volumétrica dessa área são esperados, uma vez que o solo é arenoso (Neossolo Regolítico), que possui teores de areia superiores a 80\%, com baixa retenção de água e elevada drenagem (ALMEIDA et al., 2015; SANTOS et al., 2012; SILVA et al., 2014).

Observa-se, ainda, quando ocorreram veranicos (períodos sem chuvas dentro da estação chuvosa), como nos períodos de 23/05 a 09/06/2014 e de 17/08 a 05/09/2014,

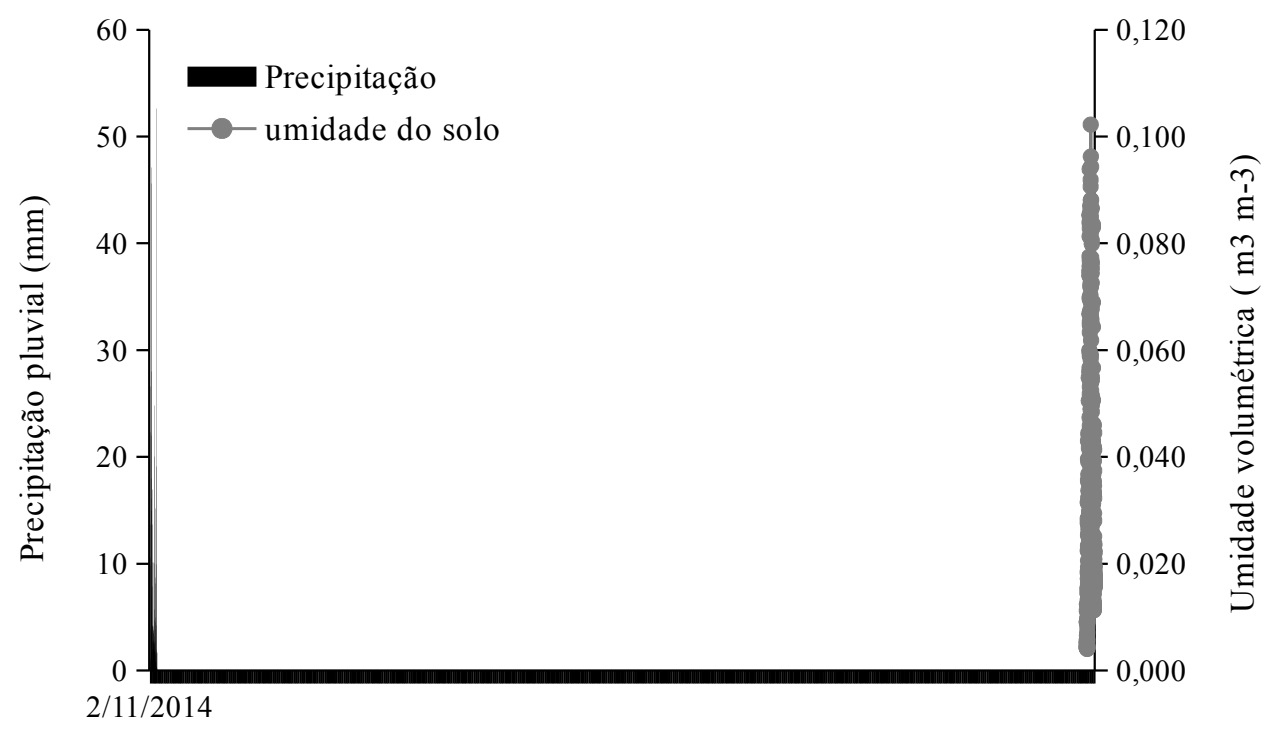

Tempo (dias)

Figura 1. Precipitação pluvial e umidade do solo em Caatinga, durante o período de 01/01/2014 e 31/12/2014 no município de São João - PE. 


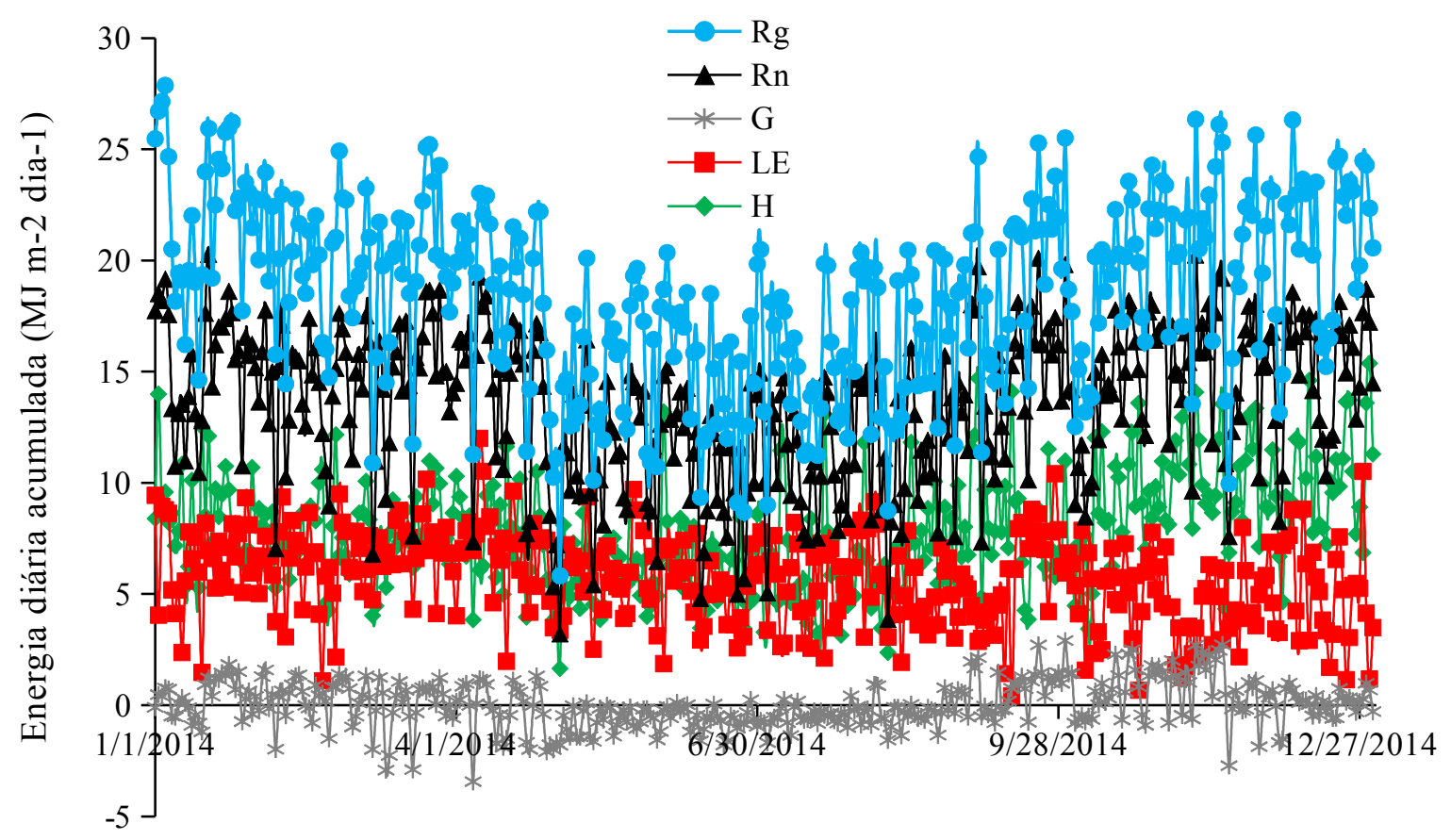

Tempo (dias)

Figura 2. Variação diária da radiação global (Rg), do saldo de radiação (Rn), dos fluxos de calor latente (LE), de calor sensível (H) e de calor no solo (G) em área de Caatinga em São João-PE, para o ano de 2014.

que a umidade do solo decresceu linearmente com a falta de chuva, sendo que aumentou novamente somente após eventos de chuvas maiores que $5 \mathrm{~mm}$. Isso indica que chuvas menores que $5 \mathrm{~mm}$ podem ter sido interceptadas pelo dossel da Caatinga. Em relação as perdas de água por interceptação vegetal, Medeiros et al. (2009), numa Caatinga preservada no semiárido do Ceará, encontraram que as mesmas podem chegar a $13 \%$ do total precipitado.

A Figura 2 mostra a variação diária dos fluxos de energia (Rn, LE, H e G) e da radiação solar global (Rg) durante o período de 01/01/2014 a 31/12/2014.

Os valores de Rg diários variaram entre 6,0 e 27,9 MJ $\mathrm{m}^{-2} \mathrm{~d}^{-1}$, com valor médio de $18,5 \pm 4,1 \mathrm{MJ} \mathrm{m}^{-2} \mathrm{~d}^{-1}$. Os maiores valores de $\mathrm{Rg}$ (acima de $18 \mathrm{MJ} \mathrm{m}^{-2} \mathrm{~d}^{-1}$ ) ocorreram no período Setembro a Março, provavelmente devido à ausência de chuvas e de nebulosidade, e os menores (abaixo de 15 MJ $\left.\mathrm{m}^{-2} \mathrm{~d}^{-1}\right)$ no período de Maio a Agosto, principalmente nos dias em que houveram precipitações e no período do inverno da região (Figura 2). Souza et al. (2015b), estudando os componentes do balanço de radiação em Caatinga preservada em Petrolina-PE, encontraram que os maiores valores de Rg ocorreram no mês de Janeiro e foram da ordem de 29,0 MJ m ${ }^{-2} \mathrm{~d}^{-1}$, com os menores valores ocorrendo nos meses de inverno, corroborando os dados encontrados nesse trabalho.

Em relação ao saldo de radiação $(\mathrm{Rn})$, observa-se que seus valores oscilaram entre 3,2 e $20,3 \mathrm{MJ} \mathrm{m}^{-2} \mathrm{~d}^{-1}$, com valor médio de 13,5 $\pm 3,4 \mathrm{MJ} \mathrm{m}^{-2} \mathrm{~d}^{-1}$. A Rn seguiu as variações sazonais da Rg, com os maiores valores de Rg ocorrendo nos períodos de maiores valores de Rg (período seco) e os menores no período mais úmido (Figura 1). Souza et al. (2015a) estudando os fluxos de energia em Caatinga na região de Petrolina-PE num ano de seca extrema (2012), encontraram que a Rn teve variações sazonais e seus valores máximo, médio e mínimo foram de 18,11 e $6 \mathrm{MJ} \mathrm{m}^{-2} \mathrm{~d}^{-1}$, respectivamente.

Os maiores valores de Rn na estação úmida (Maio a Agosto de 2014) podem estar associados com os maiores valores de Rg nessa estação, uma vez que existe uma relação consistente entre os valores de Rn e Rg para a região semiárida nordestina, como encontrado por Teixeira et al. (2008), em Caatinga na cidade de Petrolina, PE. Oliveira et al. (2006), estudaram os fluxos de energia em Caatinga, também encontraram que a variação sazonal da Rn acompanhou a variação sazonal da Rg, com os menores valores de Rn ocorrendo no mês de Julho. Já em estudo dos fluxos de energia em Caatinga preservada e Caatinga degradada na cidade de Campina Grande-PB, Borges et al. (2016), encontraram que a Rn também apresentou sazonalidade, com os maiores valores observados no período de Outubro a Janeiro e os menores no período de Junho a Agosto, corroborando com os dados encontrados nesse trabalho.

Em relação ao fluxo de calor no solo (G), verificou-se que o mesmo apresentou os menores valores dentre todos os componentes do balanço de energia (Figura 2). $0 \mathrm{G} \mathrm{va-}$ riou de $-3,44$ a 2,91 $\mathrm{MJ} \mathrm{m}^{-2} \mathrm{~d}^{-1}$, com média de $0,04 \mathrm{MJ} \mathrm{m}^{-2}$ $\mathrm{d}^{-1}$. Os menores valores de $\mathrm{G}$, do mesmo modo que a Rg e a Rn, ocorreram nos períodos em que se teve precipitação 
pluvial, e os valores mais elevados ocorreram nos períodos de seca. Cerca de $52 \%$ dos valores diários do $\mathrm{G}$ foram negativos, indicando perda de energia da superfície do solo para a atmosfera, como também encontrado por Souza et al. (2015a), em Petrolina-PE. Os valores de $G$ desta pesquisa estão dentro dos normalmente encontrados em áreas de Caatinga (OLIVEIRA et al., 2006; TEIXEIRA et al., 2008).

Analisando o balanço de energia em áreas de Caatingas preservada e degradada, Borges et al. (2016) encontraram maiores variação e valores de $G$ nas áreas de caatinga degradada, possivelmente devido a uma maior exposição desta a incidência da Rg, o que faz com que ocorra um maior aquecimento do solo e consequentemente maior $\mathrm{G}$.

Em relação ao fluxo de calor latente (LE), observou-se que seus valores variaram de 0,4 a $12,0 \mathrm{MJ} \mathrm{m}^{-2} \mathrm{~d}^{-1}$, com valor médio de $5,7 \mathrm{MJ} \mathrm{m}^{-2} \mathrm{~d}^{-1}$. Esses valores médios de LE estão um pouco acima dos obtidos por Teixeira et al. (2008), que estudando o balanço de energia em Caatinga encontraram valores médios anuais de 4,62 e 2,67 $\mathrm{MJ} \mathrm{m}^{-2} \mathrm{~d}^{-1}$, nos anos de 2004 e 2005, respectivamente. No entanto, estão bem acima do valor médio encontrado por Souza et al. (2015a), que foi de apenas $0,77 \mathrm{MJ} \mathrm{m}^{-2} \mathrm{~d}^{-1}$, em Caatinga na cidade de Petrolina-PE. As diferenças entre os valores médios anuais de LE são devido, provavelmente, a disponibilidade de água no sistema solo-planta-atmosfera, uma vez que na pesquisa de Souza et al. (2015a), somente ocorreram 92,0 $\mathrm{mm}$ em todo ano de 2012, e nesta pesquisa a precipitação pluvial foi de $754,3 \mathrm{~mm}$.

Com relação ao fluxo de calor sensível (H) foi observado que o mesmo foi maior que LE durante a maior parte do período de estudo, com seus valores variando de 1,6 a 15,4 $\mathrm{MJ} \mathrm{m}{ }^{-2} \mathrm{~d}^{-1}$, com valor médio de $7,8 \mathrm{MJ} \mathrm{m}^{-2} \mathrm{~d}^{-1}$. Resultados semelhantes de $\mathrm{H}$ foram encontrados por outros autores que mediram os fluxos de energia em região semiárida (OLIVEIRA et al., 2006; TEIXEIRA et al., 2008). Contudo, valores mais elevados de H foram obtidos por Souza et al. (2015a), em ano de seca extrema numa Caatinga em Petrolina-PE. De acordo com Oliveira et al. (2006), de modo geral, regiões semiáridas como o bioma Caatinga, apresentam baixas taxas de LE, pois a maior parte da energia disponível à superfície (Rn-G) é liberada na forma de calor sensível.

As variações de LE e H (Figura 2) correspondem com as variações da precipitação pluvial e da umidade volumétrica do solo (Figura 1), já que nos períodos de maior disponibilidade hídrica do solo ocorrem os maiores valores de LE. No entanto, isso ocorreu em poucos dias do ano, sendo que numa escala anual o fluxo de calor sensível (H) foi muito maior que o fluxo de calor latente (LE).

A partição da energia disponível pode ser melhor analisada por meio das relações LE/Rn e H/Rn. Numa escala anual a razão $\mathrm{LE} / \mathrm{Rn}$ foi de 0,421 , indicando que $42,1 \%$ do saldo de radiação $(\mathrm{Rn})$ foi usado para o processo de evapotranspiração (ET); já a razão H/Rn foi de 0,576, ou seja,
$57,6 \%$ do Rn foi usado para o aquecimento do ar. Nossos resultados estão de acordo com os obtidos por Teixeira et al. (2008), que encontraram razão LE/Rn anual variando de 0,25 a 0,40 e razão H/Rn de 0,49 a 0,64 em área de Caatinga preservada em Petrolina, PE. As diferenças foram devidas a disponibilidade hídrica do solo.

Todas as variáveis mencionadas nas Figuras 1 e 2, quais sejam, precipitação pluvial umidade do solo, Rg, Rn, LE, H e $G$, seguiram um ciclo sazonal. Nos períodos mais úmidos (meses de Maio a Agosto), com menores Rg, Rn, $\mathrm{H} \mathrm{e}$ $G$, ocorreram os maiores valores de umidade do solo e LE, devido aos maiores valores de precipitação pluvial. $O$ inverso ocorreu nos meses de Janeiro a Março e de Setembro a Dezembro. Essas variações sazonais também foram encontradas por outros pesquisadores (BORGES et al., 2016; OLIVEIRA et al., 2006; SOUZA et al., 2015a; TEIXEIRA et al., 2008).

A variação diária da precipitação pluvial e da evapotranspiração (ET) da Caatinga é mostrada na Figura 3. Observa-se, do mesmo modo que a umidade do solo (Figura 1) e os fluxos de energia (Figura 2), que a ET seguiu as variações da precipitação pluvial. A ET total para o período de 365 dias (01/01 a 31/12/2014) foi de $852 \mathrm{~mm}$ e a ET média foi de 2,3 $\mathrm{mm} \mathrm{d}^{-1}$. A ET diária variou de 0,2 a $4,9 \mathrm{~mm} \mathrm{~d}^{-1}$, com os maiores valores nos períodos de maior umidade do solo (Figura 1) e com maior Rn (Figura 2); o inverso ocorreu nos períodos em que a umidade do solo foi baixa e/ou o Rn também foi baixo.

Esses valores de ET da Caatinga estão bem acima dos obtidos por Souza et al. (2015a), que mediram a ET em Caatinga no Semiárido pernambucano encontrando valores que oscilaram de 0,5 a 3,0 $\mathrm{mm} \mathrm{d}^{-1}$. As diferenças encontradas entre nossa pesquisa e as desses autores deve ser devida a quantidade de água no sistema, uma vez que a pesquisa de Souza et al. (2015a) foi desenvolvida num ano de seca extrema, com apenas $92 \mathrm{~mm}$ de precipitação pluvial, enquanto a nossa teve 754,3 $\mathrm{mm}$. Além disso, nossa pesquisa fica localizada na região agreste, a qual possui condições climáticas mais favoráveis que a região semiárida de Pernambuco.

Observa-se, ainda, na Figura 3, que a ET total da Caatinga foi $852 \mathrm{~mm}$, enquanto a precipitação total foi 754,3 $\mathrm{mm}$, ou seja, a ET foi $97,7 \mathrm{~mm}$ maior que a precipitação. Esse maior valor de ET deve ter ocorrido, provavelmente, devido a retirada de água pelo sistema radicular da Caatinga de camadas mais profundas do solo. Nesse sentido, em estudo da profundidade efetiva do sistema radicular (profundidade onde se encontra pelo menos $80 \%$ das raízes) de uma Caatinga no estado do Ceará, Pinheiro et al. (2013) encontraram que as raízes da Caatinga podem chegar até a $0,78 \mathrm{~m}$.

Outras pesquisas que mediram o balanço de energia e a ET em culturas também encontraram que a ET foi maior 


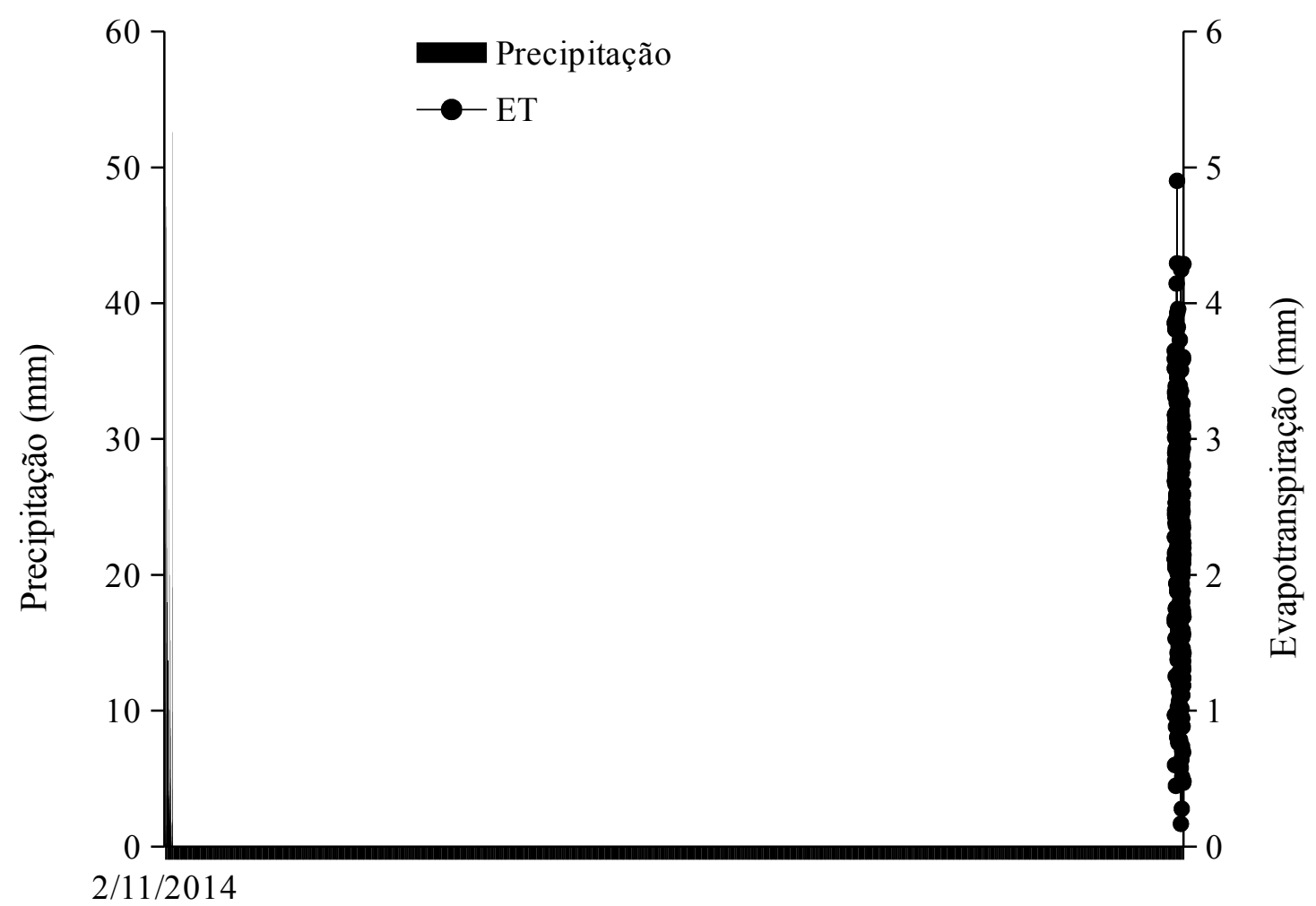

Tempo (dias)

Figura 3. Variação diária da precipitação pluvial e da evapotranspiração (ET) de Caatinga em São João-PE, para o ano de 2014.

que a precipitação. Por exemplo, Gondim et al. (2015) mediram os componentes do balanço de energia em pastagem de capim braquiária durante 01 ano (2011), no mesmo solo e região desta pesquisa, e encontraram que a ET total da pastagem foi $543,8 \mathrm{~mm}$, enquanto que a precipitação total nesse período foi de 523,7 $\mathrm{mm}$, ou seja, a ET foi $20,1 \mathrm{~mm}$ maior que a precipitação pluvial.

\section{Conclusões}

A maior parte do saldo de radiação foi usada no fluxo de calor sensível e foi controlada pela variação sazonal da precipitação pluvial e da umidade volumétrica do solo. A evapotranspiração total (852 $\mathrm{mm})$ e média $\left(2,3 \mathrm{~mm} \mathrm{~d}^{-1}\right) \mathrm{da}$ Caatinga apresentou valores elevados, em decorrência da boa disponibilidade de água no solo no período estudado. 0 método do balanço de energia - razão de Bowen mostrou-se adequado para analisar as variações diárias e sazonais dos fluxos de energia e da evapotranspiração da Caatinga.

\section{Agradecimentos}

Os autores agradecem ao Instituto Nacional de Ciência e Tecnologia: Observatório Nacional da Dinâmica da Água e do Carbono no Bioma Caatinga (INCT-ONDACBC), aprovado na chamada pública MCTI/CNPq/CAPES/FAPS $N^{\circ}$ 16/2014 Programa INCT, financiado pela Facepe, Capes (processo $\left.\mathrm{N}^{\circ} 88887.136339 / 2017-00\right)$ e CNPq (processo No 465764/2014-2).

\section{Referências}

ALMEIDA, A. V. D. L. et al. Atributos Físicos, Macro e Micromorfológicos de Neossolos Regolíticos no Agreste Meridional de Pernambuco. Revista Brasileira de Ciência do Solo, Viçosa, v. 39, n. 5, p. 1235-1246, 2015.

APAC - Agência Pernambucana de Águas e Clima. http://www.apac. pe.gov.br >. 5 Abr. 2016.

BALDOCCHI, D. D. Assessing the eddy covariance technique for evaluating carbon dioxide exchange rates of ecosystems: past, present and future. Global Change Biology, Oxford, v. 9, n. 4, p. 479-492, 2003.

BARRETO, C. E. A. G.; WENDLAND, E.; MARCUZZO, F. F. N. Estimativa da evapotranspiração a partir de variação de nível estático de aquífero. Engenharia Agrícola, Jaboticabal, v. 29, n. 1, p. 52-61, 2009.

BORGES, C. K. et al. Variação sazonal dos fluxos de energia em áreas de caatinga preservada e degradada no Nordeste Brasileiro. Revista Brasileira de Geografia Física, Recife, v. 9, n. 5, p. 1313-1321, 2016.

CIRILO, J. A. Políticas públicas de recursos hídricos para o semi-árido. Estudos Avançados, São Paulo, v. 22, n. 63, p. 61-82, 2008.

GONDIM, P. S. S. et al. Environmental control on water vapour and energy exchanges over grasslands in semiarid region of Brazil. Revista Brasileira de Engenharia Agrícola e Ambiental, Campina Grande, v. 19, n. 1, p. 3-8, 2015.

LIMA, J. R. S. et al. Balanço de energia e evapotranspiração de feijão caupi sob condições de sequeiro. Revista Ciência Agronômica, Fortaleza, v. 42, n. 1, p. 65-74, 2011.

LIMA, J. R. S. et al. Balanço de energia em um solo cultivado com feijão caupi no brejo paraibano. Revista Brasileira de Engenharia Agrícola e Ambiental, Campina Grande, v. 9, n. 4, p. 527-534, 2005. 
LIMA, J. R. S. et al. Seasonal and interannual variations of evapotranspiration, energy exchange, yield and water use efficiency of castor grown under rainfed conditions in northeastern Brazil. Industrial Crops and Products, Amsterdam, v. 50, n. 1, p. 203-211, 2013.

MEDEIROS, P. H. A.; ARAÚJO, J. C.; BRONSTERT, A. Interception measurements and assessment of Gash model performance for a tropical semi-arid region. Revista Ciência Agronômica, Fortaleza, v. 40, p. 165174. 2009.

MENEZES, R. S. C. et al. Biogeochemical cycling in terrestrial ecosystems of the Caatinga Biome. Brazilian Journal of Biology, São Carlos, v. 72, n. 3, p. 643-653, 2012.

OLIVEIRA, M. B. L. et al. Trocas de energia e fluxo de carbono entre a vegetação de caatinga e atmosfera no nordeste brasileiro. Revista Brasileira de Meteorologia, São José dos Campos, v. 21, n. 3b, p. 159165, 2006.

PINHEIRO E. A. R.; COSTA C. A.G.; ARAÚJO J. C. Effective root depth of the Caatinga biome. Journal of Arid Environments, Amsterdam, v. 89, p. 1-4, 2013.

SAMPAIO, E. V. S. B. et al. Residual N and P fertilizer effect and fertilizer recovery on intercropped and sole-cropped corn and beans in semiarid northeast Brazil. Nutrient Cycling and Agroecosystems, Amsterdam, v. 70, p. 1-11, 2004.
SANTOS, J. C. B. et al. Caracterização de Neossolos Regolíticos da região semiárida de Pernambuco. Revista Brasileira de Ciência do Solo, Viçosa, v. 36, n. 3, p. 683-695, 2012.

SILVA, R.A.B. et al. Balanço hídrico em Neossolo regolítico cultivado com braquiária (Brachiaria Decumbens Stapf). Revista Brasileira de Ciência do Solo, Viçosa, v. 38, n. 1, 147-157, 2014.

SOUZA, L. S. B. et al. Balanço de energia e controle biofísico da evapotranspiração na Caatinga em condições de seca intensa. Pesquisa Agropecuária Brasileira, Brasília, v. 50, n. 8, p. 627-636, 2015a.

SOUZA, L. S. B. et al. Balanço de radiação em ecossistema de Caatinga preservada durante um ano de seca no semiárido Pernambucano. Revista Brasileira de Geografia Física, Recife, v. 8, n.1, p. 41-55, 2015 b.

TEIXEIRA, A. H. de C. et al. Analysis of energy fluxes and vegetation-atmosphere parameters in irrigated and natural ecosystems of semi-arid Brazil. Journal of Hydrology, Amsterdam, v. 362, p. 110127, 2008.

TODD, R. W.; EVETT, S. R.; HOWELL, T. A. The Bowen ratio energy balance method for estimating latent heat flux of irrigated alfalfa evaluated in a semi-arid, advective environment. Agricultural and Forest Meteorology, Amsterdam, v. 103, n. 04, p 335-348, 2000.

WEVER, L. A.; FLANAGAN, L. B.; CARLSON, P.J. Seasonal and interannual variation in evapotranspiration, energy balance and surface conductance in a northern temperate grassland. Agricultural and Forest Meteorology, Amsterdam, v. 112, 31-49, 2002.

\footnotetext{
REFERENCIAÇÃO LIMA, J. R. de S.; OlIVEIRA, C. L. de; BARBOSA, A. da C.; ANTONINO, A. C. D.; SOUZA, E. S. de. Variação sazonal dos fluxos de energia e evapotranspiração em caatinga no agreste Pernambucano. Agrometeoros, Passo Fundo, v.25, n.1, p.113-120, 2017.
}

Declaração: os trabalhos estão sendo publicados nesse número de AGROMETEOROS (v.25, n.1, ago 2017) conforme foram aceitos pelo XX Congresso Brasileiro de Agrometeorologia, realizado de 14 a 18 de agosto de 2017, em Juazeiro, BA e Petrolina, PE, sem revisão editorial adicional da revista. 


\title{
Seasonal variation in energy fluxes and evapotranspiration over caatinga in agreste region of Pernambuco State, Brazil
}

\author{
José Romualdo de Sousa Lima ${ }^{1(*)}$, Cássio Lopes de Oliveira ${ }^{2}$, Amós da Cruz Barbosa ${ }^{3}$, Antonio Celso Dantas Antonino ${ }^{4}$, \\ Eduardo Soares de Souza ${ }^{5}$ \\ ${ }^{1}$ Professor Associado, UAG/UFRPE, Garanhuns, PE, romualdo.lima@ufrpe.br \\ ${ }^{2}$ Estudante de Agronomia, UAG/UFRPE, Garanhuns, PE, cassio.agronomo@yahoo.com \\ ${ }^{3}$ Estudante de Agronomia, UAG/UFRPE, Garanhuns, PE, amos.barbosa@outlook.com \\ ${ }^{4}$ Professor Titular, DEN/UFPE, Recife, PE, acdantonino@gmail.com \\ ${ }^{5}$ Professor Associado, UAST/UFRPE, Serra Talhada, PE, edu_souza_pe@yahoo.com.br \\ ${ }^{(*)}$ Corresponding author
}

\section{ARTICLE INFO}

Article history:

Received 16 June 2017

Accepted 10 August 2017

Index terms:

semiarid

climate changes

soil moisture

\section{ABSTRACT}

The energy fluxes, i.e, net radiation (Rn) and the latent heat (LE), sensible heat (H) and soil heat $(\mathrm{G})$ fluxes and the evapotranspiratio (ET) have a great importance in aspects various of time and climate and they are associated with the carbon fluxes. The eddy covariance method is one of the most used to measure these fluxes, however it is still expensive. Thus, the ideal is to use a cheaper method and also accurate, such as energya balance - Bowen ratio. The objective of this study was to assess the seasonal variation of Rn, LE, H, G and ET under preserved Caatinga, at town of São João, PE, by using the Bowen ratio method. For that, the area was instrumented with a micrometeorological tower of $8 \mathrm{~m}$ height in the center of the area, containing a rain gauge, a net radiometer and sensors for measuring air temperature and relative humidity at two levels above the canopy of Caatinga. In addition, a soil moisture sensor and a soil heat flux plate, in $5.0 \mathrm{~cm}$ deep, were installed. The sensible heat flux $(\mathrm{H})$ was the main consumer of the available energy $(\mathrm{Rn})$ and was controlled by seasonal variation of the rainfall and the soil moisture. The total $(852 \mathrm{~mm})$ and average $(2.3 \mathrm{~mm} \mathrm{~d}-1)$ values for ET in Caatinga were high, due to the good availability of water in the soil in the studied period.

(C) 2017 SBAgro. All rights reserved.

CITATION

LIMA, J. R. de S.; OLIVEIRA, C. L. de; BARBOSA, A. da C.; ANTONINO, A. C. D.; SOUZA, E. S. de. Variação sazonal dos fluxos de energia e evapotranspiração em caatinga no agreste Pernambucano. Agrometeoros, Passo Fundo, v.25, n.1, p.113-120, 2017.

Disclaimer: papers are published in this issue of AGROMETEOROS (v. 25, n.1, aug 2017) as accepted by the XX Congresso Brasileiro de Agrometeorologia, held August 14-18, 2017 in Juazeiro, Bahia and Petrolina, Pernambuco, Brazil, without further revision by editorial board. 